Florida International University

FIU Digital Commons

Biomolecular Sciences Institute: Faculty

Publications

College of Arts, Sciences \& Education

$1-1-2015$

\title{
Targeting bacterial topoisomerase I to meet the challenge of finding new antibiotics
}

Yuk-Ching Tse-Dinh

Department of Chemistry \& Biochemistry, Florida International University, ytsedinh@fiu.edu

Follow this and additional works at: https://digitalcommons.fiu.edu/biomolecular_fac

\section{Recommended Citation}

Tse-Dinh YC. Targeting bacterial topoisomerase I to meet the challenge of finding new antibiotics. Future Med Chem. 2015;7(4):459-71. doi: 10.4155/fmc.14.157. PMID: 25875873; PMCID: PMC4415981.

This work is brought to you for free and open access by the College of Arts, Sciences \& Education at FIU Digital Commons. It has been accepted for inclusion in Biomolecular Sciences Institute: Faculty Publications by an authorized administrator of FIU Digital Commons. For more information, please contact dcc@fiu.edu. 


\title{
Targeting bacterial topoisomerase I to meet the challenge of finding new antibiotics
}

\author{
Yuk-Ching Tse-Dinh ${ }^{1,2}$ \\ Yuk-Ching Tse-Dinh: ytsedinh@fiu.edu \\ ${ }^{1}$ Department of Chemistry \& Biochemistry, Florida International University, 11200 SW 8 St, \\ Miami, FL 33199, USA \\ 2Biomolecular Sciences Institute, Florida International University, 11200 SW 8 St, Miami, FL \\ 33199, USA Tel.: +1 305348 4956, Fax: +1 3053483772
}

\begin{abstract}
Resistance of bacterial pathogens to current antibiotics has grown to be an urgent crisis. Approaches to overcome this challenge include identification of novel targets for discovery of new antibiotics. Bacterial topoisomerase I is present in all bacterial pathogens as a potential target for bactericidal topoisomerase poison inhibitors. Recent efforts have identified inhibitors of bacterial topoisomerase I with antibacterial activity. Additional research on the mode of action and binding site of these inhibitors would provide further validation of the target and establish that bacterial topoisomerase I is druggable. Bacterial topoisomerase I is a potentially high value target for discovery of new antibiotics. Demonstration of topoisomerase I as the cellular target of an antibacterial compound would provide proof-of-concept validation.
\end{abstract}

\section{Type IA topoisomerase as a novel bactericidal target}

\section{The need for new antibiotics}

The emergence of bacterial pathogens resistant to current antibiotics is an increasingly urgent worldwide public health problem [1]. New drugs are needed for therapeutic regimens that can be effective for treatment of multidrug-resistant and extensively drug-resistant tuberculosis [2]. Drug-resistant gram-negative pathogens including Pseudomonas aeruginosa [3] and Acinetobacter baumannii [4] are becoming more prevalent in the hospital settings. Methicillin-resistant Staphylococcus aureus strains have developed resistance to vancomycin, traditionally a drug of last resort [5]. New antibiotics that can be effective against multiple bacterial pathogens, including the ESKAPE pathogens

(Enterococcus faecium, S. aureus, Klebsiella pneumoniae, Acinetobacter baumannii, $P$.

\footnotetext{
(C) 2015 Future Science Ltd

For reprint orders, please contact reprints@ future-science.com

No writing assistance was utilized in the production of this manuscript.

Financial \& competing interests disclosure

Research on this topic was supported by grants to Y-C Tse-Dinh from National Institutes of Health (GM054226, AI069313) and TB Alliance. The authors have no other relevant affiliations or financial involvement with any organization or entity with a financial interest in or financial conflict with the subject matter or materials discussed in the manuscript apart from those disclosed.
} 
aeruginosa and Enterobacter species) [6], are much needed [7]. Focusing on a novel target different from the targets involved in mode of action of current antibiotics could provide new drugs that might avoid the resistance mechanisms for current drugs.

\section{Bacterial topoisomerase I as a novel bactericidal target}

At least one type IA topoisomerase enzyme can be found in every bacterium [8]. This class of topoisomerases regulates DNA topology by first generating a break on a single strand of DNA with a tyrosine nucleophile at the active site, forming a covalent complex with the cleaved DNA via a 5'-phosphotyrosine linkage [9]. The DNA is rejoined after the movement of DNA strands necessary for the DNA topological change. The type IA topoisomerase function is essential for resolving topological barriers that require single-strand DNA passage [10]. Type IIA topoisomerases, in contrast, break and rejoin a double strand of DNA [11]. Bacterial type IIA topoisomerases (gyrase and topoisomerase IV) are highly utilized targets for antibacterial drugs, including the quinolones and other chemical compounds [1214]. The widely prescribed quinolones are highly effective bactericidal agents not only because the cellular functions of the type IIA topoisomerases are inhibited by the quinolone action but also due to the fact that stabilized ternary complex formed by gyrase or topoisomerase IV with cleaved DNA plus the quinolone triggers chromosomal fragmentation and bacterial cell death [12]. Inhibitors that can cause cell death by trapping the covalent intermediate after DNA cleavage by topoisomerases are known as topoisomerase poison inhibitors [15]. Unlike catalytic enzyme inhibitors that must inhibit nearly all of the cellular activity to be effective, topoisomerase poison inhibitors need to trap only a relatively small subset of the cellular topoisomerase molecules as covalent complex to trigger cell death [16]. Numerous anticancer drugs of clinical importance have been found to act as poison inhibitors against human type IIA topoisomerases, as well human type IB topoisomerase [15]. An interfacial mechanism has been proposed for these topoisomerase poison inhibitors with the inhibitors binding to both enzyme and DNA in the ternary complex [17]. Type IA topoisomerase present in all bacterial pathogens could be a novel target for bactericidal antibiotics. Bacteria that have more than one type IA topoisomerases, for example, Helicobacter pylori and Escherichia coli, would provide opportunity for dual targeting of the homologous type IA enzymes to decrease the rate of drug resistance.

\section{Bacterial cell death from dominant lethal topoisomerase I mutations that inhibit DNA religation}

Since type IA topoisomerases covalent complex accumulation would lead to single-strand breaks in chromosomal DNA instead of the double-strand breaks expected from accumulation of type IIA topoisomerase covalent complexes, it needs to be verified that the bacterial topoisomerase I covalent complex accumulation can initiate the bacterial cell death pathway. This was accomplished following random mutagenesis of recombinant bacterial topoisomerase I and identification of topoisomerase I mutants that can induce the SOS DNA repair system in response to the DNA damage $[18,19]$. The D111N mutation at the first aspartate of the DxD motif in the TOPRIM domain of E. coli topoisomerase I was found to be extremely lethal as a result of loss of DNA rejoining activity due to the absence of the negatively charged carboxylate side chain required for divalent $\mathrm{Mg}^{2+}$ binding [20]. Small molecules that interfere with $\mathrm{Mg}^{2+}$ binding at the active site could potentially function as 
type IA topoisomerase poison inhibitors [19]. A strictly conserved arginine residue proximal to the active site tyrosine was also found to the critical for DNA rejoining [21].

\section{Potential processing activities for bacterial topoisomerase covalent complexes}

The bacterial topoisomerase I mutants that accumulate the covalent cleavage complex allow genetic studies of the processing and repair pathways of the topoisomerase covalent complex in E. coli. The initial single-strand DNA break may be converted by RuvAB and RecG into a double-strand DNA break [22]. SOS response is thus induced to activate the RecBCD pathway for repair of the double-strand break [23]. It is not clear which bacterial proteolytic or nucleolytic activities might be responsible for removing the covalently bound topoisomerase protein, but $\mathrm{SbcCD}$ nuclease is a likely candidate $[24,25]$. Nuclease homologues of SbcCD have been shown to remove covalently bound topoisomerases from DNA in eukaryotes [26]. TDP1 and TDP2 tyrosyl-DNA phosphodiesterases have been shown to cleave the phosphotyrosine linkage following proteolytic processing of the topoisomerase covalent complex in eukaryotes [15,27-28]. TDP1 and TDP2 inhibitors are sought in screening assays for combination chemotherapy to improve response to anticancer topoisomerase poison inhibitors [29,30]. Homologs of TDP1 and TDP2 or enzymes of similar functions remain to be identified in bacteria.

\section{Essential functions of bacterial type IA topoisomerase Topoisomerase I function in stress response and virulence}

Bacterial type IA topoisomerases participate in many essential cellular processes including replication, transcription, recombination and repair [31]. For bacteria with more than one type IA topoisomerase encoded in the genome, it might be possible to isolate mutants missing one of the type IA topoisomerase activities under laboratory growth conditions. However, as illustrated by the studies of E. coli $\triangle$ topA mutants, absence of the topA encoded topoisomerase I function results in hypersensitivity to antibiotics as well as high temperature and oxidative stress [32-34]. This is likely due to the requirement of topoisomerase I relaxation activity for removal of transcription-driven supercoiling at loci with high rate of transcription of genes needed for adaption and survival in the natural or host environments [35]. Hypernegative supercoiling and R-loop formation during transcription elongation would prevent synthesis of the stress response genes, resulting in loss of viability [36]. While either bacterial topoisomerase I and topoisomerase III can probably meet the requirement of type IA topoisomerase activity in chromosome segregation [37,38], the relatively slow rate of topoisomerase III catalytic activity for relaxation of negative supercoils [39] would likely not meet the demands for rapid transcription elongation in the host environment. Interestingly, even though $\mathrm{H}$. pylori has two type IA topoisomerases that are annotated as TopA because these enzymes have higher degree of sequence homology to known bacterial topoisomerase I than topoisomerase III, results from transposon mutagenesis experiment indicate that the longer form of the H.pylori TopA is nevertheless essential, suggesting that these two TopA enzymes have nonoverlapping functions for $H$. pylori, which may include adaptation in the acidic environment $[40,41]$. 
Bacterial type IA topoisomerases can also have essential roles in pathogenic virulence through the regulation of transcription and recombination. Deletion of E. coli topA gene reduced the expression of FooB, a regulator of fimbriae F165(1) in pathogenic E. coli [42]. Inactivation of Pectobacterium atrosepticum topoisomerase III $\beta$ has been shown to result in reduced virulence due to hyperexcision of the pathogenicity island HAI2 [43]. Additional type IA topoisomerases were found encoded by the pXO1 virulence plasmid of Bacillus anthracis [44], and cystic fibrosis associated isolates of P. aeruginosa [45].

\section{Type IA topoisomerase function required for viability}

In organisms with only one type IA topoisomerase, the topA gene encoding topoisomerase I is essential for viability, as demonstrated for Mycobacterium tuberculosis [46] and Streptomyces coelicolor [47]. These topoisomerase I enzymes might be responsible for functions associated with topoisomerase III in other bacteria that have both of these type IA topoisomerases, including resolving intermediates in chromosome segregation and recombination [31]. While E. coli topoisomerase III is known to interact with RecQ helicase to control recombination [48], RecQ homologs are not found in Mycobacteria. Helicases that may interact with Mycobacterium topoisomerase I in replication and recombination functions remain to be identified. For consideration of another potential function for bacterial topoisomerase I enzyme, human type IA topoisomerase III $\beta$ has been shown to be an RNA topoisomerase required for regulation of mRNA metabolic reactions [49,50]. E. coli topoisomerase III can also act as an RNA topoisomerase [51], but it remains unclear if a bacterial RNA topoisomerase activity might be utilized to modify cellular RNA structures.

While type IA topoisomerase poison inhibitors remain a highly desirable class of inhibitors for discovery as broad-spectrum bactericidal antibiotics leads, catalytic inhibitors of bacterial topoisomerase I could potentially be useful for targeting organisms that absolutely require topoisomerase I activity for viability, for example, in treatment of tuberculosis. The requirement of topoisomerase I function in transcription during stress response further enhances the potential of synergistic efficacy from topoisomerase I inhibitors if used along with other antibiotics in combination antibacterial therapy. The identification of a small molecule inhibitor with topoisomerase I as the proven mode of antibacterial action and the required selectivity would validate bacterial topoisomerase I as a druggable target.

\section{Assays development for high-throughput screening}

\section{Cell-based SOS induction assay}

Recombinant bacterial topoisomerase I with mutations that mimic the effect of topoisomerase poison inhibitors for accumulation of covalent complex with cleaved DNA were initially identified based on the resulting SOS response induced in E. coli [18]. Trapping of gyrase covalent complex by quinolones was also known to induce the SOS response in E. coli [52]. A high-throughput screening (HTS) assay aiming to identify type IA topoisomerase poison inhibitors was therefore based on induction of the E. coli SOS response [53]. Luciferase activity from plasmid encoded $\operatorname{din} D$ 1:luxCADBE fusion [54] was utilized as reporter of SOS induction via transcription from the $\operatorname{din} D 1$ promoter. Synthesis of the recombinant luxCADBE gene products from Photorhabdus luminescens can generate 
luminescence without addition of luciferase substrate. The HTS assay attempted to identify compounds that could induce greater luciferase signal when wild-type recombinant Yersinia pestis topoisomerase I was over-expressed in comparison to when an active site mutant of $Y$. pestis topoisomerase I was overexpressed. Three bacterial topoisomerase I poison inhibitors were identified from HTS screening [53]. These compounds had no effect on type IB vaccinia topoisomerase I cleavage, but two of the compounds derived from phenanthrene and fluorene were later found to be poison inhibitors for mitochondrial type IIA topoisomerases in Trypanosoma brucei [55]. The SOS induction assay may select for DNA intercalators. While many topoisomerase poison inhibitors are DNA intercalators that can act as interfacial inhibitors, DNA intercalators are also likely to have a high tendency of affecting activities of more than one class of DNA topoisomerases.

\section{Cell-based supercoiling-sensitive reporter assay}

Inhibition of bacterial gyrase or topoisomerase I activity in vivo is expected to alter global chromosomal DNA supercoiling [56]. E. coli promoters for gyrA and topA gene respond reciprocally to changes in supercoiling in the homeostatic regulation of supercoiling $[57,58]$. In the design of an assay for in vivo bacterial DNA supercoiling, the ratio of the specific activity of ptopA to the specific activity of pgyrA monitored by luciferase reporter plasmids was defined as quotient of supercoiling (Qsc) [59]. The Qsc value increases in the presence of topA mutation and decreases in the presence of gyrase mutation or catalytic inhibitors of gyrase such as novobiocin. Plant extracts prepared from Angelica archangelica and Ruta graveolens were found to decrease the Qsc value [59]. Decrease in Qsc was also observed in the presence of two coumarin compounds present in these plant extracts. Compounds or natural product extracts that increase the Qsc values as expected from inhibition of topoisomerase I activity were not discussed. This in vivo assay could potentially be used to identify compounds that can inhibit either the supercoiling activity of gyrase or the relaxation activity of topoisomerase I in bacterial cells.

\section{Topoisomerase catalytic assays based on supercoiling-sensitive DNA structures}

Assays that are based on increased efficiency of intermolecular triplex formation for negatively supercoiled DNA have been designed for identification of inhibitors of either the supercoiling activity of DNA gyrase, or relaxation activity of topoisomerases [60,61]. These assays can be applied in HTS of inhibitors of bacterial topoisomerase I enzymes. Maxwell et al. developed assays based on capture of a plasmid containing triplex-forming sequence by an oligonucleotide immobilized on microtiter plate well surface, and subsequent detection of the plasmid captured by triplex formation with fluorescence dye [61,62]. A homogeneous assay based on the same principle but does not require immobilization, filtration or washing steps measured the fluorescence anisotropy of oligonucleotide labeled with fluorescent dye following intermolecular triplex formation with plasmid [60]. This homogeneous assay has been shown to be applicable for measuring the relaxation activity of $E$. coli topoisomerase I. However, results of HTS utilizing such triplex-based assays on bacterial topoisomerase I have not been reported.

Cruciform extrusion from inverted repeat sequence in plasmids is also dependent on negative supercoiling [63]. This dependence on negative supercoiling was utilized to 
develop a high-throughput real-time fluorescence-based assay for detection of inhibitors of the supercoiling activity of bacterial DNA gyrase [64]. In principle, this assay could also be adapted to monitor the removal of negative supercoils by bacterial topoisomerase I, but such data have not been reported for this catalytic assay.

\section{Enzyme-based assay for increase in DNA cleavage}

The assays based on triplex formation and cruciform extrusion cannot identify specifically topoisomerase poison inhibitors. Previous studies on quinolones showed that these type II topoisomerase poison inhibitors can inhibit bacterial cell growth at concentrations significantly lower than the $\mathrm{IC}_{50}$ concentrations for inhibition of the overall supercoiling catalytic activity [65] because initiation of bactericidal pathway by stabilized topoisomerase cleavage complex on the chromosome does not require that most of the cellular topoisomerase activity be inhibited. Nalidixic acid, the first known quinolone poison inhibitor of $E$. coli gyrase, requires concentration of $100 \mu \mathrm{M}$ or more for $50 \%$ inhibition of the gyrase supercoiling activity $[65,66]$. Inhibitors with such high $\mathrm{IC}_{50}$ values would most likely not be selected as hits to be followed up in HTS. Therefore, potential topoisomerase poison inhibitors might not be captured with the HTS assays based on overall catalytic activity. It is thus desirable to have a HTS assay that can detect inhibition of DNA rejoining by bacterial topoisomerase I to identify poison inhibitors that shift the DNA cleavagereligation equilibrium toward DNA cleavage.

A fluorescence-based assay has been developed to detect increase in DNA cleavage products from bacterial topoisomerase I. This assay utilizes a single-stranded oligonucleotide that forms a stem-loop structure with a preferred cleavage site for bacterial topoisomerase I in the single-stranded loop region indicated by an arrow (Figure 1). Fluorescence signal from a fluorophore placed at the $5^{\prime}$-end is limited by a quencher present at the $3^{\prime}$-end. Upon heating, fluorescence signal increases from melting of the secondary structure. Destabilization of the structure from increase in DNA cleavage by bacterial topoisomerase I would lead to a higher fluorescence signal. This was demonstrated with the greater than fivefold increase in fluorescence from the G116S mutation in E. coli topoisomerase I known to lead to deficiency in DNA religation [18,67]. The oligonucleotide for the HTS assay was initially designed for $Y$. pestis topoisomerase I for identifying inhibitors that might be useful in countering $Y$. pestis as a bioterror threat. E. coli and $Y$. pestis topoisomerase I are nearly identical in enzymatic properties, including preference in DNA cleavage site selection. Following assay development using the NCI Diversity Set I for identifying suitable positive control, it was found that compound NSC28086 at $0.25 \mathrm{mM}$ concentration can result in greater than fivefold increase in fluorescence signal over background signal in the presence of DMSO. $Z^{\prime}$ factor of $0.7-0.8$ can be achieved on different days during pilot screening at the NERCE/NSRB screening center [67].

Counterscreening in the absence of enzyme was carried out to eliminate compounds that due to either autofluorescence or effect on DNA structure, could result in the same degree of fluorescence increase with or without enzyme present. The use of a fluorophore reporter with emission in the higher wavelength range such as CAL Fluor Red 610 minimizes the overlap of fluorescence emission wavelengths between the assay reporter and most library compounds. There was fluorescence increase from adding NSC20806 to the oligonucleotide 
substrate alone but $25 \%$ greater increase in fluorescence could be observed in the presence of enzyme. This HTS assay was carried out at Broad Institute. From 356,421 compounds screened in the primary assay, 229 active compounds were identified [68].

\section{Newly identified bacterial topoisomerase I inhibitors}

A number of bacterial topoisomerase I inhibitors have been described in an earlier review [19]. Bacterial topoisomerase I inhibitors reported in publications since then will be discussed here. Available data on these inhibitors are summarized in Table 1.

\section{Phenanthrene compounds}

A phenanthrene compound stephenanthrine was among the bacterial topoisomerase I poison inhibitors identified in the HTS based on induction of SOS response in E. coli [53]. More recently, following semi-synthesis and evaluation of alkaloids from bolden, two phenanthrenes seconeolitsine and $N$-methyl-seconeolitsine (Compounds 1, 2 in Figure 2) were found to inhibit Streptococcus pneumoniae topoisomerase I relaxation and cell growth at concentrations of approximately $17 \mu \mathrm{M}$ [69]. Hypernegative supercoiling of plasmid DNA extracted from S. pneumoniae following treatment with seconeolitsine is consistent with inhibition of cellular topoisomerase I relaxation activity by the compound. Attempts to isolate mutants resistant to these inhibitors were not successful but overproduction of recombinant $S$. pneumoniae topoisomerase I was observed to provide some degree of protection against growth inhibition by seconeolitsine in support of topoisomerase I being an in vivo target [69]. These phenanthrenes are DNA intercalators but their inhibition effect on the $S$. pneumoniae topoisomerase I relaxation activity is distinct from the intercalator effect of ethidium bromide, which did not appear to inhibit the bacterial topoisomerase I relaxation activity. Inhibition of topoisomerase I relaxation activity by these phenanthrenes was enhanced by preincubation of the inhibitors with enzyme prior to addition of DNA. It was proposed from docking studies using a molecular model of S. pneumoniae topoisomerase I structure that the inhibitors may be occupying the nucleotide-binding site of the enzyme through cation $-\pi$ interactions to prevent subsequent DNA substrate binding. This proposed mechanism remains to be validated by x-ray-based structures of the enzyme-inhibitor complex. Toxicological studies suggested that seconeolitsine and $N$-methyl-seconeolitsine at $30 \mu \mathrm{M}$ did not affect human neutrophil viability [69]. $N$-methyl-seconeolitsine has been used in a later study to trigger increased level of DNA supercoiling and activation of iron transport gene $f a t D$ transcription in $S$. pneumoniae demonstrating the potential utility of this compound as a probe for type IA topoisomerase function [75].

\section{Bisbenzimidazoles}

Fluorescent stains Hoechst 33342 (Compound 3, Figure 3) and Hoechst 33258 (Compound 4, Figure 3) are DNA minor groove binders that have been known to act as mammalian DNA topoisomerase I poison inhibitors [76]. New bisbenzimidazole analogues of these Hoechst dyes that can selectively target bacterial DNA topoisomerase I over human topoisomerases have been synthesized and characterized [70-73]. 3,4-Dimethoxyphenyl bisbenzimidazole [70,71] (DMA, Compound 5 in Figure 3) as well as DPA 153 [72] with a terminal alkyne substitution (Compound 6 in Figure 3) represent independently discovered 
bisbenzimidazole analogues that inhibit $E$. coli topoisomerase I much more strongly than $E$. coli DNA gyrase, human topoisomerase I and human topoisomerase IIa. The $\mathrm{IC}_{50}$ for $E$. coli topoisomerase I inhibition by DMA was found to be $3.8 \mu \mathrm{M}$ [70], very similar to the $\mathrm{IC}_{50}$ value of $2.5 \mu \mathrm{M}$ for DPA 153 [72]. There was evidence that DMA can act as a poison inhibitor for $E$. coli topoisomerase I, increasing the level of DNA cleavage products [70]. DMA was effective for growth inhibition of clinical isolates of $E$. coli, with no apparent cytotoxic effect on mammalian cells [70,71]. The antibacterial activity of DMA increased slightly when E. coli topoisomerase I is overexpressed, consistent with cell killing initiated by trapping of the topoisomerase I covalent complex [70]. Additional bisbenzimidazoles analogues have been synthesized more recently for biological assays [73]. Bisbenzimidazole 12b, 5-(4-Propylpiperazin-1-yl)-2-[2'-(4-ethoxyphenyl)-5'-benzimidazolyl]benzimidazole (Compound 7 in Figure 3$)$ has improved $\mathrm{IC}_{50}$ value $(2 \mu \mathrm{M})$ over DMA and the lowest MIC values $(0.1-8 \mu \mathrm{g} / \mathrm{ml})$ among the latest analogues when tested against different $E$. coli strains, including drug-resistant clinical isolates and water-borne $E$. coli strains. Direct interaction of this compound with $E$. coli topoisomerase I can be demonstrated by isothermal titration calorimetry, with Ka value of $6.8 \times 10^{6} \mathrm{M}^{-1}$ and $\Delta \mathrm{G}$ value of $-10.84 \mathrm{kcal} / \mathrm{ml}$ [73]. Furthermore, in vivo efficacy was demonstrated with mouse systemic infection model and neutropenic thigh model.

\section{Natural products as dual inhibitors of type IA \& type IIA topoisomerases}

A number of natural product extracts were included in the pilot screening carried out at NERCE/NSRB screening center with the fluorescence-based assay for bacterial topoisomerase I poison inhibitors targeting $Y$. pestis topoisomerase I [67]. Lichen Hypotrachyna sp. extract was among the natural product extracts found to result in $>60 \%$ increase in fluorescence signal versus the DMSO negative control. The extract also had antibacterial activity against $E$. coli strain BAS3023 with imp4213 mutation that increases permeability for small molecules [77]. Fractionation of the lichen Hypotrachyna sp. (Lecanorales, Parmeliaceae) from Costa Rica resulted in identification of the depside anziaic acid (Compound 8 in Figure 4) as a bacterial topoisomerase I poison inhibitor [67]. Anziaic acid is not active against $E$. coli with wild-type membrane permeability, but can inhibit growth of gram-positive Bacillus subtilis with MIC of $6 \mu \mathrm{g} / \mathrm{ml}(14 \mu \mathrm{M})$. The $0.9 \mu \mathrm{M}$ concentration required to observe increase in DNA cleavage product formed by $E$. coli topoisomerase I is significantly lower than the $\mathrm{IC}_{50}$ value of $19 \mu \mathrm{M}$ for inhibition of the relaxation activity as expected for topoisomerase poison inhibitor. Anziaic acid can inhibit type IIA topoisomerase activities (E. coli DNA gyrase and human topoisomerase IIa) with similar $\mathrm{IC}_{50}$ values, but inhibition against type IB human topoisomerase I is much weaker. Anziaic acid could potentially interfere with enzyme-divalent ion interactions at the active site [67]. The poison inhibitor effect of anziaic acid on human topoisomerase IIa might partly account for its cytotoxicity against human pulmonary artery endothelial cells (CC50 = $21 \mu \mathrm{M})$. Total synthesis of anziaic acid and its analogues was carried out subsequently for preliminary structure-activity analysis of topoisomerase Inhibition and antibacterial activity [78]. The results showed that the dimeric scaffold and the free carboxylate of the compound are essential for topoisomerase Inhibition. The negatively charged carboxylate may be required for divalent ion interaction in the topoisomerase active site but antibacterial assays showed that the carboxylate is not required for antibacterial activity, suggesting that there is 
an unknown antibacterial mode of action in addition to topoisomerase Inhibition. The lipophilic n-pentyl alkyl substituents augment both topoisomerase inhibition and antibacterial activity [78].

Dual inhibition of both type IIA bacterial DNA topoisomerases, gyrase, and topoisomerase IV has been utilized to reduce the emergence of drug-resistant bacterial pathogens [12,79]. For bacterial pathogens with only one type IIA topoisomerase, such as M. tuberculosis, dual inhibition of both type IA topoisomerase I and type IIA DNA gyrase could provide the same advantage of potentially reducing drug resistance frequency. Type IA and type IIA topoisomerases share many mechanistic and structural similarities, including the formation of a 5'-phosphotyrosine covalent linkage by active site tyrosine in CAP-like domains and requirement of divalent ions bound by TOPRIM domains [80,81]. In addition to anziaic acid, 2,4-diiodoemodin (Compound 9 in Figure 4), a haloemodin derived from the bioactive natural product emodin also exhibits dual inhibition against DNA gyrase and bacterial topoisomerase I [74]. Complete inhibition of these bacterial topoisomerase activities can be achieved with concentrations of 30-90 $\mu \mathrm{M}$, while human topoisomerase I and IIa were not inhibited. The MIC of 2,4-diiodoemodin against $S$. aureus $(2 \mu \mathrm{g} / \mathrm{ml})$ is comparable to vancomycin, with strong activities against other Gram-positive bacteria, including drugresistant isolates, also observed [74]. S. aureus-induced keratitis can be cured in rabbit model, demonstrating the therapeutic efficacy of this compound. The antibacterial spectrum can be expended into Gram-negative bacteria if penetration through the bacterial outer membrane is assisted by polymyxin B nonapeptide [74].

The known type IIA human topoisomerase II poison inhibitor, m-AMSA was reported to inhibit the relaxation activity of M. tuberculosis and M. smegmatis topoisomerase I in follow up of virtual screening on a homology model of M. tuberculosis topoisomerase I [82]. DNA cleavage was stimulated up to 1.7 fold, and m-AMSA can inhibit the growth of these mycobacteria. However, at least part of the growth inhibition may be due to DNA intercalation and inhibition of other DNA enzymes. In previous screening using the triplex formation based assay, m-AMSA and related compounds were also identified as inhibitors of the relaxation activity type IIB Methanosarcina mazei topoisomerase VI [83]. Utilization of this class of acridine-based compounds as specific type IA topoisomerase Inhibitors would require greater selectivity. The same caution of target selectivity also applies to the alkaloid ungeremine found to inhibit mammalian as well as bacterial type I and type II topoisomerases [84], and the other polyphenolic compounds discussed earlier in this review, as such compounds are frequent hits in many biochemical assays.

\section{Protein inhibitors of bacterial topoisomerase I}

In addition to small molecules, certain proteins have also been shown to inhibit the relaxation activity of bacterial topoisomerase I. These include the $E$. coli $\mathrm{Tn} 5$ transposase discussed in the previous review [19] and M. tuberculosis MazF homolog Rv1495 [85]. Rv1495 can inhibit the DNA cleavage activity of M. tuberculosis topoisomerase I. The negative interplay of the direct physical interaction between the two proteins also resulted in the inhibition of the mRNA cleavage activity of Rv1495 by the C-terminal domain of $M$. tuberculosis topoisomerase I. Overexpression of Rv1495 or its $N$-terminal fragment in $M$. 
smegmatis can inhibit cell growth [85]. This result is significant for the potential modulation of bacterial topoisomerase I activity and by toxin-antitoxin system in relationship to dormancy regulation. Interaction between bacterial topoisomerase I and toxin protein may possibly be found also in other bacteria, including $E$. coli [11]. It is plausible that small molecules could be effective antibiotics by mimicking the action of protein inhibitors of bacterial topoisomerase I. Monoclonal antibodies that can inhibit the relaxation activity of M. tuberculosis and M. smegmatis topoisomerase I have been described [86,87]. These antibodies can act with different mechanisms, including stimulation of DNA cleavage [86] as well as enhancing the closing of the DNA gate after DNA cleavage [87]. These antibodies could be valuable for the design of novel bacterial topoisomeraseI inhibitors.

\section{Conclusion \& future perspective}

The true validation of bacterial topoisomerase I as a druggable target for antibiotics discovery would require the identification of an antibacterial compound that involves topoisomerase I in its major mode of action. The effect of topoisomerase I expression level on antibacterial potency can provide some quick indication of whether topoisomerase I may be one of the cellular targets for growth inhibition. Overexpression of topoisomerase I should have protective effect against catalytic inhibitors as demonstrated for seconeolitsine [69], but should conversely sensitize the bacteria to the action of a poison inhibitor, as suggested for DMA [70]. It should be cautioned that the role of the relaxation activity of topoisomerase I in regulation of global DNA supercoiling and transcription elongation during stress response may be significant for degree of sensitivity to DNA intercalators or other stress-inducing agents. To demonstrate convincingly that the antibacterial mode of action requires direct interactions between the compound and cellular topoisomerase $\mathrm{I}$, it is still very much desirable to isolate resistant mutants with mutations in the topA gene, and then to show that the compound MIC can be affected when the identified topA mutation is introduced into the original drug sensitive genetic background. This has not been carried out for any bacterial topoisomerase I inhibitors.

Testing of selectivity of bacterial topoisomerase I inhibitors against human topoisomerases has so far only involved type IB and type IIA human topoisomerases. However, there are two type IA topoisomerases in human, TOP $3 a$ and TOP $3 \beta$, that should be evaluated in any future development of bacterial topoisomerase Inhibitors into drug leads. There are many examples of US FDA-approved drugs that target either bacterial type IIA topoisomerases as antibiotics or human type IIA topoisomerases as anticancer drugs, so it should be possible to optimize the structures of type IA topoisomerase Inhibitors for selectivity against either bacterial or human type IA topoisomerases. Mammalian TOP3a is essential for embryonic development, and its DNA decatenation activity plays an important role in genomic stability [88]. Human TOP $3 \beta$ has RNA topoisomerase activity that affects neurodevelopment and has been associated with mental disorders [49,50]. Selective small molecule inhibitors for these human type IA topoisomerase activities could be very useful research tools for experimental investigations with implications for human diseases.

Attempts have been made to model the interactions between the newly discovered bacterial topoisomerase I inhibitors with the target enzyme $[69,73,82]$ to support the proposals of 
possible mechanisms of action or to carry out virtual screening. Structure-based drug design and identification of druggable sites on the enzyme would benefit greatly from an experimentally determined structure of the topoisomerase-inhibitor or topoisomeraseDNA-inhibitor complex. To limit potential mutagenicity or cytotoxicity, it would be advantageous to occupy the drug-binding sites with molecular scaffolds that are not strong DNA binders. Structural models of S. pneumoniae and M. tuberculosis topoisomerase I have been built based on homology with available topoisomerase I structures [69,82]. Additional three-dimensional structures of topoisomerase I enzymes of the targeted pathogens would improve the potential accuracy of in silico results. While it is expected that the DNA cleavage-religation equilibrium of type IA topoisomerases is primarily controlled by the Nterminal toroid like domain, the $\mathrm{C}$-terminal domain is also required for the overall relaxation activity $[89,90]$, and may affect the cleavage-religation equilibrium through indirect allosteric effects [20]. Elucidation of the C-terminal domain structures should further aid the drug discovery effect targeting bacterial topoisomerase I.

\section{Key terms}

Type IA

topoisomerase

Topoisomerase poison

Bactericidal

antibiotics

Bacterial

topoisomerase I

High-throughput screening
Subfamily of topoisomerases that catalyze change in DNA topology by breaking and rejoining a single strand of DNA via a $5^{\prime}$-phosphotyrosine covalent intermediate in a divalent ion dependent mechanism

Inhibitors of topoisomerases that kill cells by increasing the level of the covalent intermediate formed between topoisomerase enzyme and cleaved DNA

Antibacterial treatment that kills bacteria

A type IA topoisomerase enzyme found in every bacterium

Automated high-throughput screening of thousands to millions of individual compounds or natural product extracts

\section{References}

Papers of special note have been highlighted as:

- of interest; $\bullet$ of considerable interest

1. Carlet J, Pulcini C, Piddock LJ. Antibiotic resistance: a geo-political issue. Clin MicroBiol Infect. 2014; 20(10):949-953. [PubMed: 25040923]

2. Gunther G. Multidrug-resistant and extensively drug-resistant tuberculosis: a review of current concepts and future challenges. Clin Med. 2014; 14(3):279-285. [PubMed: 24889573]

3. Bodro M, Sabe N, Tubau F, et al. Extensively drug-resistant Pseudomonas aeruginosa bacteremia in solid organ transplant recipients. Transplantation. 2014; 99(3):616-622. [PubMed: 25119130]

4. Hischebeth GT, Wimmer MD, Molitor E, Seifert H, Gravius S, Bekeredjian-Ding I. Multidrug resistant Acinetobacter baumannii reaches a new frontier: prosthetic hip joint infection. Infection. 201410.1007/s15010-014-0661-x 
5. Gardete S, Tomasz A. Mechanisms of vancomycin resistance in Staphylococcus aureus. J Clin Invest. 2014; 124(7):2836-2840. [PubMed: 24983424]

6. Boucher HW, Talbot GH, Bradley JS, et al. Bad bugs, no drugs: no ESKAPE! An update from the Infectious Diseases Society of America. Clin Infect Dis. 2009; 48(1):1-12. [PubMed: 19035777]

7. Bassetti M, Merelli M, Temperoni C, Astilean A. New antibiotics for bad bugs: where are we? Ann Clin MicroBiol Antimicrob. 2013; 12:22-0711-0712-22. [PubMed: 23984642]

8. Forterre P, Gadelle D. Phylogenomics of DNA topoisomerases: their origin and putative roles in the emergence of modern organisms. Nucleic Acids Res. 2009; 37(3):679-692. [PubMed: 19208647]

9. Tse YC, Kirkegaard K, Wang JC. Covalent bonds between protein and DNA. Formation of phosphotyrosine linkage between certain DNA topoisomerases and DNA. J Biol Chem. 1980; 255(12):5560-5565. [PubMed: 6155377]

10. Wang JC. Cellular roles of DNA topoisomerases: a molecular perspective. Nat Rev Mol Cell Biol. 2002; 3(6):430-440. [PubMed: 12042765]

11. Chen SH, Chan NL, Hsieh TS. New mechanistic and functional insights into DNA topoisomerases. Annu Rev BioChem. 2013; 82:139-170. Comprehensive and informative review of mechanisms and functions of the different families of DNA topoisomerases. [PubMed: 23495937]

12. Aldred KJ, Kerns RJ, Osheroff N. Mechanism of quinolone action and resistance. Biochemistry. 2014; 53(10):1565-1574. [PubMed: 24576155]

13. Mitscher LA. Bacterial topoisomerase Inhibitors: quinolone and pyridone antibacterial agents. Chem Rev. 2005; 105(2):559-592. [PubMed: 15700957]

14. Mayer C, Janin YL. Non-quinolone inhibitors of bacterial type IIA topoisomerases: a feat of bioisosterism. Chem Rev. 2014; 114(4):2313-2342. [PubMed: 24313284]

15••. Pommier Y. Drugging topoisomerases: lessons and challenges. ACS Chem Biol. 2013; 8(1):8295. Comprehensive and informative review on topoisomerases as drug targets and interfacial mechanism for topoisomerase Inhibitors. [PubMed: 23259582]

16. Aedo S, Tse-Dinh YC. Isolation and quantitation of topoisomerase complexes accumulated on Escherichia coli chromosomal DNA. Antimicrob Agents Chemother. 2012; 56(11):5458-5464. [PubMed: 22869559]

17. Pommier Y, Leo E, Zhang H, Marchand C. DNA topoisomerases and their poisoning by anticancer and antibacterial drugs. Chem Biol. 2010; 17(5):421-433. [PubMed: 20534341]

18••. Cheng B, Shukla S, Vasunilashorn S, Mukhopadhyay S, Tse-Dinh YC. Bacterial cell killing mediated by topoisomerase I DNA cleavage activity. J Biol Chem. 2005; 280(46):38489-38495. First report of bacterial cell death from accumulation of bacterial topoisomerase I cleavage product to validate the enzyme as a novel target for bactericidal antibiotics. [PubMed: 16159875]

19. Tse-Dinh YC. Bacterial topoisomerase I as a target for discovery of antibacterial compounds. Nucleic Acids Res. 2009; 37(3):731-737. [PubMed: 19042977]

20. Cheng B, Annamalai T, Sorokin E, Abrenica M, Aedo S, Tse-Dinh YC. Asp-to-Asn substitution at the first position of the DxD TOPRIM motif of recombinant bacterial topoisomerase I is extremely lethal to E. coli. J Mol Biol. 2009; 385(2):558-567. [PubMed: 19013470]

21. Narula G, Annamalai T, Aedo S, et al. The strictly conserved Arg-321 residue in the active site of Escherichia coli topoisomerase I plays a critical role in DNA rejoining. J Biol Chem. 2011; 286(21):18673-18680. [PubMed: 21478161]

22. Sutherland JH, Tse-Dinh YC. Analysis of RuvABC and RecG involvement in the Escherichia coli response to the covalent topoisomerase-DNA complex. J Bacteriol. 2010; 192(17):4445-4451. [PubMed: 20601468]

23. Sutherland JH, Cheng B, Liu IF, Tse-Dinh YC. SOS induction by stabilized topoisomerase IA cleavage complex occurs via the RecBCD pathway. J Bacteriol. 2008; 190(9):3399-3403. [PubMed: 18310346]

24. Connelly JC, de Leau ES, Leach DR. Nucleolytic processing of a protein-bound DNA end by the E. coli SbcCD (MR) complex. DNA Repair (Amst). 2003; 2(7):795-807. [PubMed: 12826280]

25. Aedo S, Tse-Dinh YC. SbcCD-mediated processing of covalent gyrase-DNA complex in Escherichia coli. Antimicrob Agents Chemother. 2013; 57(10):5116-5119. [PubMed: 23917316] 
26. Hartsuiker E, Neale MJ, Carr AM. Distinct requirements for the Rad32(Mre11) nuclease and Ctp1(CtIP) in the removal of covalently bound topoisomerase I and II from DNA. Mol Cell. 2009; 33(1):117-123. [PubMed: 19150433]

27. Pouliot JJ, Yao KC, Robertson CA, Nash HA. Yeast gene for a Tyr-DNA phosphodiesterase that repairs topoisomerase I complexes. Science. 1999; 286(5439):552-555. [PubMed: 10521354]

28. Ledesma FC, El Khamisy SF, Zuma MC, Osborn K, Caldecott KW. A human 5'-tyrosyl DNA phosphodiesterase that repairs topoisomerase-mediated DNA damage. Nature. 2009; 461(7264): 674-678. [PubMed: 19794497]

29. Marchand C, Huang SY, Dexheimer TS, et al. Biochemical assays for the discovery of TDP1 inhibitors. Mol Cancer Ther. 2014; 13(8):2116-2126. [PubMed: 25024006]

30. Raoof A, Depledge P, Hamilton NM, et al. Toxoflavins and deazaflavins as the first reported selective small molecule inhibitors of tyrosyl-DNA phosphodiesterase II. J Med Chem. 2013; 56(16):6352-6370. [PubMed: 23859074]

31. Vos SM, Tretter EM, Schmidt BH, Berger JM. All tangled up: how cells direct, manage and exploit topoisomerase function. Nat Rev Mol Cell Biol. 2011; 12(12):827-841. [PubMed: 22108601]

32. Tse-Dinh YC. Increased sensitivity to oxidative challenges associated with topA deletion in Escherichia coli. J Bacteriol. 2000; 182(3):829-832. [PubMed: 10633122]

33. Qi H, Menzel R, Tse-Dinh YC. Increased thermosensitivity associated with topoisomerase I deletion and promoter mutations in Escherichia coli. FEMS MicroBiol Lett. 1999; 178(1):141146. [PubMed: 10483733]

34-. Liu IF, Sutherland JH, Cheng B, Tse-Dinh YC. Topoisomerase I function during Escherichia coli response to antibiotics and stress enhances cell killing from stabilization of its cleavage complex. J Antimicrob Chemother. 2011; 66(7):1518-1524. Results supporting the value of bacterial topoisomerase I as a drug target due to its role in stress response required for survival of bacteria in response to antibiotic treatment and host defense. [PubMed: 21486853]

35. Liu LF, Wang JC. Supercoiling of the DNA template during transcription. Proc Natl Acad Sci USA. 1987; 84(20):7024-7027. [PubMed: 2823250]

36. Cheng B, Rui S, Ji C, et al. RNase H overproduction allows the expression of stress-induced genes in the absence of topoisomerase I. FEMS MicroBiol Lett. 2003; 221(2):237-242. [PubMed: 12725933]

37. Usongo V, Tanguay C, Nolent F, Bessong JE, Drolet M. Interplay between type 1A topoisomerases and gyrase in chromosome segregation in Escherichia coli. J Bacteriol. 2013; 195(8):1758-1768. [PubMed: 23396913]

38. Usongo V, Drolet M. Roles of Type 1A topoisomerases in genome maintenance in Escherichia coli. PLoS Genet. 2014; 10(8):e1004543. [PubMed: 25102178]

39. Terekhova K, Marko JF, Mondragon A. Studies of bacterial topoisomerases I and III at the singlemolecule level. BioChem Soc Trans. 2013; 41(2):571-575. [PubMed: 23514156]

40. Suerbaum S, Brauer-Steppkes T, Labigne A, Cameron B, Drlica K. Topoisomerase I of Helicobacter pylori: juxtaposition with a flagellin gene (flaB) and functional requirement of a fourth zinc finger motif. Gene. 1998; 210(1):151-161. [PubMed: 9524255]

41. Dong Q, Hyde D, Herra C, et al. Identification of genes regulated by prolonged acid exposure in Helicobacter pylori. FEMS MicroBiol Lett. 2001; 196(2):245-249. [PubMed: 11267787]

42. Tessier MC, Graveline R, Crost C, et al. Effects of DNA supercoiling and topoisomerases on the expression of genes coding for F165(1), a P-like fimbriae. FEMS MicroBiol Lett. 2007; 277(1): 28-36. [PubMed: 17986081]

43. Vanga BR, Butler RC, Toth IK, Ronson CW, Pitman AR. Inactivation of PbTopo III beta causes hyper-excision of the Pathogenicity Island HAI2 resulting in reduced virulence of Pectobacterium atrosepticum. Mol MicroBiol. 2012; 84(4):648-663. [PubMed: 22524709]

44. Koehler TM. Bacillus anthracis physiology and genetics. Mol Aspects Med. 2009; 30(6):386-396. [PubMed: 19654018]

45. Head NE, Yu H. Cross-sectional analysis of clinical and environmental isolates of Pseudomonas aeruginosa: biofilm formation, virulence, and genome diversity. Infect Immun. 2004; 72(1):133144. [PubMed: 14688090] 
46•. Ahmed W, Menon S, Godbole AA, Karthik PV, Nagaraja V. Conditional silencing of topoisomerase I gene of Mycobacterium tuberculosis validates its essentiality for cell survival. FEMS MicroBiol Lett. 2014; 353(2):116-123. Demonstration that topoisomerase I function in Mycobacterium tuberculosis is essential for cell survival to support the potential development of catalytic inhibitors of Mycobacterium tuberculosis topoisomerase I into new TB drug leads. [PubMed: 24593153]

47. Szafran M, Skut P, Ditkowski B, et al. Topoisomerase I (TopA) is recruited to ParB complexes and is required for proper chromosome organization during Streptomyces coelicolor sporulation. J Bacteriol. 2013; 195(19):4445-4455. [PubMed: 23913317]

48. Harmon FG, DiGate RJ, Kowalczykowski SC. RecQ helicase and topoisomerase III comprise a novel DNA strand passage function: a conserved mechanism for control of DNA recombination. Mol Cell. 1999; 3(5):611-620. [PubMed: 10360177]

49. Xu D, Shen W, Guo R, et al. Top3beta is an RNA topoisomerase that works with fragile X syndrome protein to promote synapse formation. Nat Neurosci. 2013; 16(9):1238-1247. [PubMed: 23912945]

50. Stoll G, Pietilainen OP, Linder B, et al. Deletion of TOP3beta, a component of FMRP-containing mRNPs, contributes to neurodevelopmental disorders. Nat Neurosci. 2013; 16(9):1228-1237. [PubMed: 23912948]

51. Wang H, Di Gate RJ, Seeman NC. An RNA topoisomerase. Proc Natl Acad Sci USA. 1996; 93(18):9477-9482. [PubMed: 8790355]

52. Phillips I, Culebras E, Moreno F, Baquero F. Induction of the SOS response by new 4-quinolones. J Antimicrob Chemother. 1987; 20(5):631-638. [PubMed: 3323160]

53. Cheng B, Liu I, Tse-Dinh YC. Compounds with antibacterial activity that enhance DNA cleavage by bacterial DNA topoisomerase I. J Antimicrob Chemother. 2007; 59:640-645. [PubMed: 17317696]

54. Van Dyk TK, Wei Y, Hanafey MK, et al. A genomic approach to gene fusion technology. Proc Natl Acad Sci USA. 2001; 98(5):2555-2560. [PubMed: 11226277]

55. Tang SC, Shapiro TA. Newly identified antibacterial compounds are topoisomerase poisons in African trypanosomes. Antimicrob Agents Chemother. 2010; 54(2):620-626. [PubMed: 20008775]

56. Zechiedrich EL, Khodursky AB, Bachellier S, et al. Roles of topoisomerases in maintaining steady-state DNA supercoiling in Escherichia coli. J Biol Chem. 2000; 275(11):8103-8113. [PubMed: 10713132]

57. Menzel R, Gellert M. Regulation of the genes for E. coli DNA gyrase: homeostatic control of DNA supercoiling. Cell. 1983; 34(1):105-113. [PubMed: 6309403]

58. Tse-Dinh YC. Regulation of the Escherichia coli DNA topoisomerase I gene by DNA supercoiling. Nucleic Acids Res. 1985; 13(13):4751-4763. [PubMed: 2991845]

59. Abu Mraheil M, Heisig A, Heisig P. An improved assay for the detection of alterations in bacterial DNA supercoiling in vivo. Pharmazie. 2013; 68(7):541-548. [PubMed: 23923635]

60. Shapiro A, Jahic H, Prasad S, et al. A homogeneous, high-throughput fluorescence anisotropybased DNA supercoiling assay. J Biomol Screen. 2010; 15(9):1088-1098. [PubMed: 20930214]

61. Maxwell A, Burton NP, O’Hagan N. High-throughput assays for DNA gyrase and other topoisomerases. Nucleic Acids Res. 2006; 34(15):e104. [PubMed: 16936317]

62. Burrell MR, Burton NP, Maxwell A. A high-throughput assay for DNA topoisomerases and other enzymes, based on DNA triplex formation. Methods Mol Biol. 2010; 613:257-266. [PubMed: 19997889]

63. Lilley DM. The inverted repeat as a recognizable structural feature in supercoiled DNA molecules. Proc Natl Acad Sci USA. 1980; 77(11):6468-6472. [PubMed: 6256738]

64. Jude KM, Hartland A, Berger JM. Real-time detection of DNA topological changes with a fluorescently labeled cruciform. Nucleic Acids Res. 2013; 41(13):e133. [PubMed: 23680786]

65. Domagala JM, Hanna LD, Heifetz CL, et al. New structure-activity relationships of the quinolone antibacterials using the target enzyme. The development and application of a DNA gyrase assay. J Med Chem. 1986; 29(3):394-404. [PubMed: 3005575] 
66. Walton L, Elwell LP. In vitro cleavable-complex assay to monitor antimicrobial potency of quinolones. Antimicrob Agents Chemother. 1988; 32(7):1086-1089. [PubMed: 2847641]

67. Cheng B, Cao S, Vasquez V, et al. Identification of anziaic acid, a lichen depside from Hypotrachyna sp., as a new topoisomerase poison inhibitor. PLoS ONE. 2013; 8(4):e60770. [PubMed: 23593306]

68. PubChem BioAssay. https://pubchem.ncbi.nlm.nih.gov/assay/assaydata.html?aid=504884\&act=act

69. Garcia MT, Blazquez MA, Ferrandiz MJ, et al. New alkaloid antibiotics that target the DNA topoisomerase I of Streptococcus pneumoniae. J Biol Chem. 2010; 286(8):6402-6413. [PubMed: 21169356]

70. Bansal S, Sinha D, Singh M, Cheng B, Tse-Dinh YC, Tandon V. 3,4-Dimethoxyphenyl bisbenzimidazole, a novel DNA topoisomerase Inhibitor that preferentially targets Escherichia coli topoisomerase I. J Antimicrob Chemother. 2012; 67(12):2882-2891. [PubMed: 22945915]

71. Bansal S, Tawar U, Singh M, Nikravesh A, Good L, Tandon V. Old class but new dimethoxy analogue of benzimidazole: a bacterial topoisomerase I inhibitor. Int J Antimicrob Agents. 2010; 35(2):186-190. [PubMed: 20006473]

72. Ranjan N, Fulcrand G, King A, et al. Selective inhibition of bacterial topoisomerase I by alkynylbisbenzimidazoles. Medchemcomm. 2014; 5(6):816-825. [PubMed: 25083189]

73. Nimesh H, Sur S, Sinha D, et al. Synthesis and biological evaluation of novel bisbenzimidazoles as Escherichia coli topoisomerase IA inhibitors and potential antibacterial agents. J Med Chem. 2014; 57(12):5238-5257. Report of a bacterial topoisomerase I inhibitor with potency and selectivity required to increase survival following infection in animal model. [PubMed: 24856410]

74. Duan F, Li X, Cai S, et al. Haloemodin as novel antibacterial agent inhibiting DNA gyrase and bacterial topoisomerase I. J Med Chem. 2014; 57(9):3707-3714. [PubMed: 24588790]

75•. Ferrandiz MJ, de la Campa AG. The fluoroquinolone levofloxacin triggers the transcriptional activation of iron transport genes that contribute to cell death in Streptococcus pneumoniae. Antimicrob Agents Chemother. 2014; 58(1):247-257. First description of the use of a small molecule type IA topoisomerase Inhibitor as a probe in vivo. [PubMed: 24145547]

76. Chen AY, Yu C, Gatto B, Liu LF. DNA minor groove-binding ligands: a different class of mammalian DNA topoisomerase I inhibitors. Proc Natl Acad Sci USA. 1993; 90(17):8131-8135. [PubMed: 7690143]

77. Braun M, Silhavy TJ. Imp/OstA is required for cell envelope biogenesis in Escherichia coli. Mol Microbiol. 2002; 45(5):1289-1302. [PubMed: 12207697]

78. Lin H, Annamalai T, Bansod P, Tse-Dinh YC, Sun D. Synthesis and antibacterial evaluation of anziaic acid and analogues as topoisomerase I inhibitors. Medchemcomm. 2013; 4(12) Epub ahead of print. 10.1039/C3MD00238A

79. Tari LW, Li X, Trzoss M, et al. Tricyclic GyrB/ParE (TriBE) inhibitors: a new class of broadspectrum dual-targeting antibacterial agents. PLoS ONE. 2013; 8(12):e84409. [PubMed: 24386374]

80. Berger JM, Fass D, Wang JC, Harrison SC. Structural similarities between topoisomerases that cleave one or both DNA strands. Proc Natl Acad Sci USA. 1998; 95(14):7876-7881. [PubMed: 9653108]

81 . Schmidt BH, Burgin AB, Deweese JE, Osheroff N, Berger JM. A novel and unified two-metal mechanism for DNA cleavage by type II and IA topoisomerases. Nature. 2010; 465(7298):641644. Proposal of type IA and type IIA topoisomerase mechanism involving divalent ions that may be the basis for dual inhibitors of type IA and type IIA topoisomerases. [PubMed: 20485342]

82. Godbole AA, Ahmed W, Bhat RS, Bradley EK, Ekins S, Nagaraja V. Inhibition of Mycobacterium tuberculosis topoisomerase I by m-AMSA, a eukaryotic type II topoisomerase poison. BioChem Biophys Res Commun. 2014; 446(4):916-920. [PubMed: 24642256]

83. Taylor JA, Mitchenall LA, Rejzek M, Field RA, Maxwell A. Application of a novel microtitre plate-based assay for the discovery of new inhibitors of DNA gyrase and DNA topoisomerase VI. PLoS ONE. 2013; 8(2):e58010. [PubMed: 23469129] 
84. Casu L, Cottiglia F, Leonti M, et al. Ungeremine effectively targets mammalian as well as bacterial type I and type II topoisomerases. Bioorg Med Chem Lett. 2011; 21(23):7041-7044. [PubMed: 22014547]

85. Huang F, He ZG. Characterization of an interplay between a Mycobacterium tuberculosis MazF homolog, Rv1495 and its sole DNA topoisomerase I. Nucleic Acids Res. 2010; 38(22):8219-8230. [PubMed: 20724443]

86. Leelaram MN, Bhat AG, Hegde SM, Manjunath R, Nagaraja V. Inhibition of type IA topoisomerase by a monoclonal antibody through perturbation of DNA cleavage-religation equilibrium. FEBS J. 2012; 279(1):55-65. [PubMed: 22008463]

87. Leelaram MN, Bhat AG, Godbole AA, Bhat RS, Manjunath R, Nagaraja V. Type IA topoisomerase Inhibition by clamp closure. FASEB J. 2013; 27(8):3030-3038. [PubMed: 23612788]

88. Bocquet N, Bizard AH, Abdulrahman W, et al. Structural and mechanistic insight into Hollidayjunction dissolution by topoisomerase III alpha and RMI1. Nat Struct Mol Biol. 2014; 21(3):261268. [PubMed: 24509834]

89. Ahmed W, Bhat AG, Leelaram MN, Menon S, Nagaraja V. Carboxyl terminal domain basic amino acids of mycobacterial topoisomerase I bind DNA to promote strand passage. Nucleic Acids Res. 2013; 41(15):7462-7471. [PubMed: 23771144]

90. Szafran MJ, Strick T, Strzalka A, Zakrzewska-Czerwinska J, Jakimowicz D. A highly processive topoisomerase I: studies at the single-molecule level. Nucleic Acids Res. 2014; 42(12):7935-7946. [PubMed: 24880688] 


\section{Executive summary}

\section{Type IA topoisomerase as a novel bactericidal target}

- Bacterial topoisomerase I is a high value target for antibacterial drug discovery that remains to be fully utilized.

- Antibiotics acting as bacterial topoisomerase I poison inhibitors have the potential to be effective against a broad spectrum of bacterial pathogens due to the presence of topoisomerase I in every bacterium.

\section{Assays development for high-throughput screening}

- High-throughput screening assays are available to identification of inhibitors of bacterial topoisomerase I overall catalytic cycle for relaxation of negative supercoils, as well as the specific step of DNA rejoining.

- It remains to be seen if these high-throughput screening assays can provide selective hits with topoisomerase I inhibition as mode of action for antibacterial efficacy.

\section{New bacterial topoisomerase I inhibitors}

- Certain bacterial topoisomerase I inhibitors derived from phenanthrenes and bisbenzimidazoles have been shown to be selective inhibitors of bacterial topoisomerase I with minimal effect on human topoisomerases and limited cytotoxicity.

- There is some evidence from effect of recombinant bacterial topoisomerase I overexpression that bacterial topoisomerase I inhibition is involved in their modes of action.

\section{Natural products inhibitors}

- Natural products represent a valuable source of molecular diversity that should be further exploited for future identification of bacterial topoisomerase I inhibitors.

- There is a potential for identification of dual inhibitors of bacterial type IA and type IIA topoisomerases.

\section{Future perspective}

- Potency and selectivity of newly identified bacterial topoisomerase I inhibitors (summarized in Table 1) may suggest promising approaches for future research.

- Antibacterial mode of action of bacterial topoisomerase I inhibitors need to be confirmed by characterization of resistant mutants.

- Study of structure-activity relationship and structure-based drug design would benefit from structures of topoisomerase-inhibitor or topoisomerase-DNAinhibitor complexes. 
- There is a great need for identification of druggable sites in the enzyme structure which can be exploited by molecular scaffolds that are not strong DNA binders. 


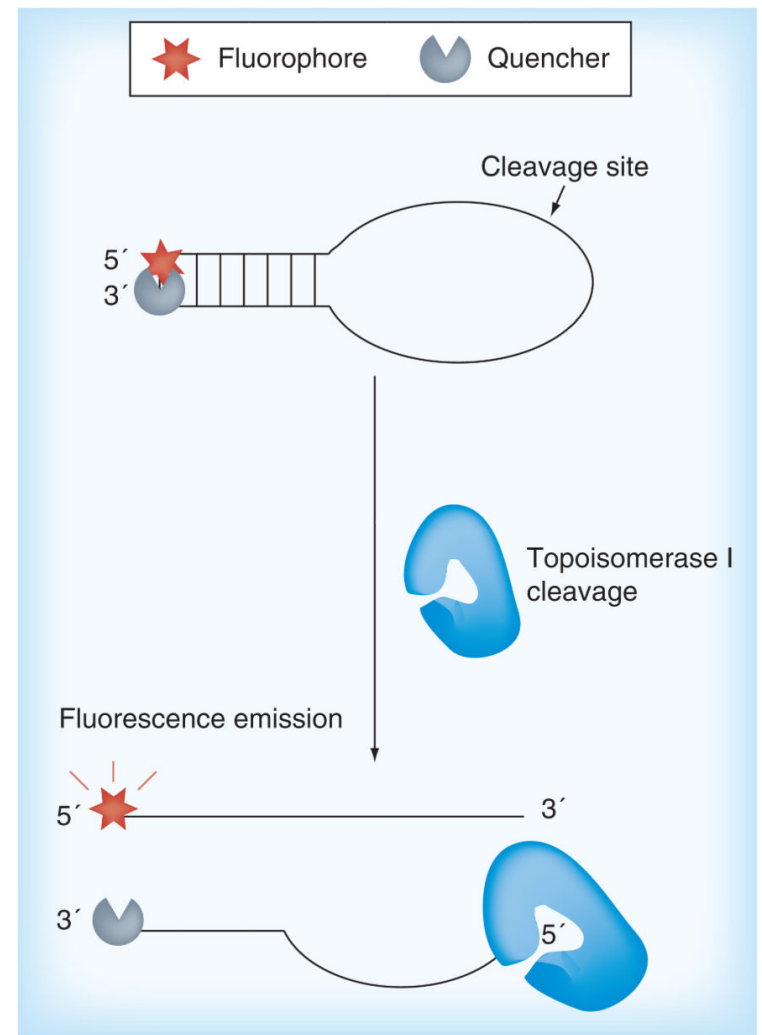

Figure 1.

Principle of fluorescence-based assay for identification of bacterial topoisomerase I poison inhibitors that can increase the level of DNA cleavage products reported as fluorescence signal increase. 


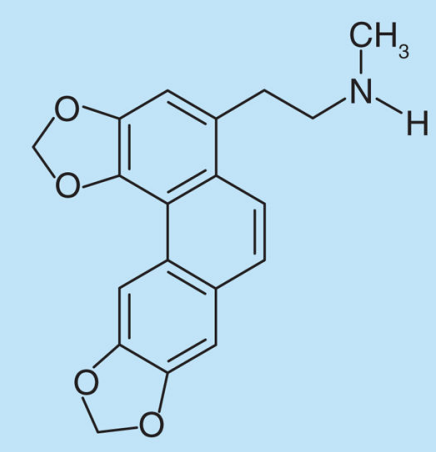

(1) Seconeolitsine

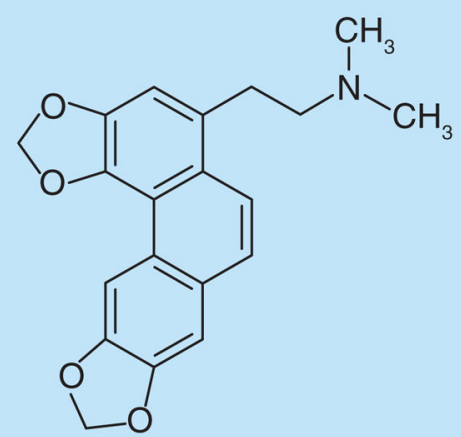

(2) N-methyl-seconeolitsine

Figure 2.

Alkaloid phenanthrenes active for inhibition of Streptococcus pneumoniae topoisomerase I relaxation and cell growth. 


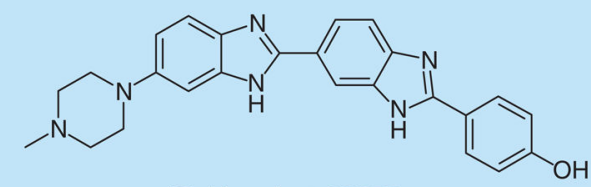

(3) Hoechst 33258
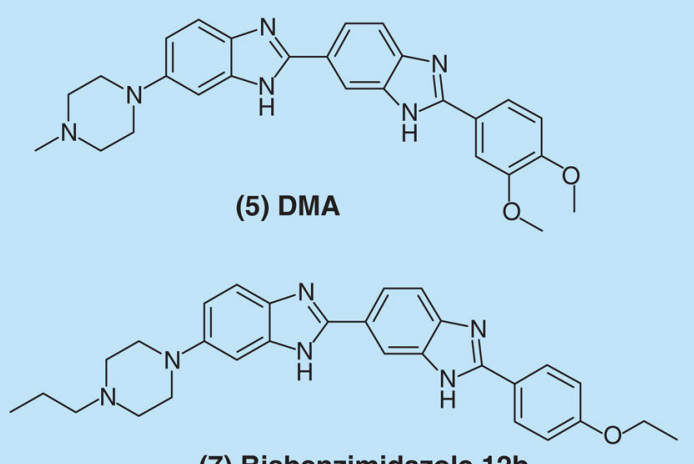

(7) Bisbenzimidazole 12b

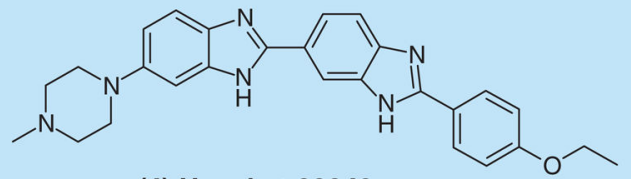

(4) Hoechst 33342

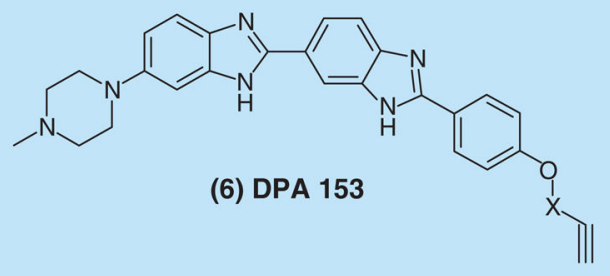

Figure 3.

Comparisons of Hoechst 33258, Hoechst 33342 with bisbenzimidazole analogues selective for bacterial topoisomerase I inhibition:DMA [70], DPA 153 [72], 12b [73].

Future Med Chem. Author manuscript; available in PMC 2016 January 01. 


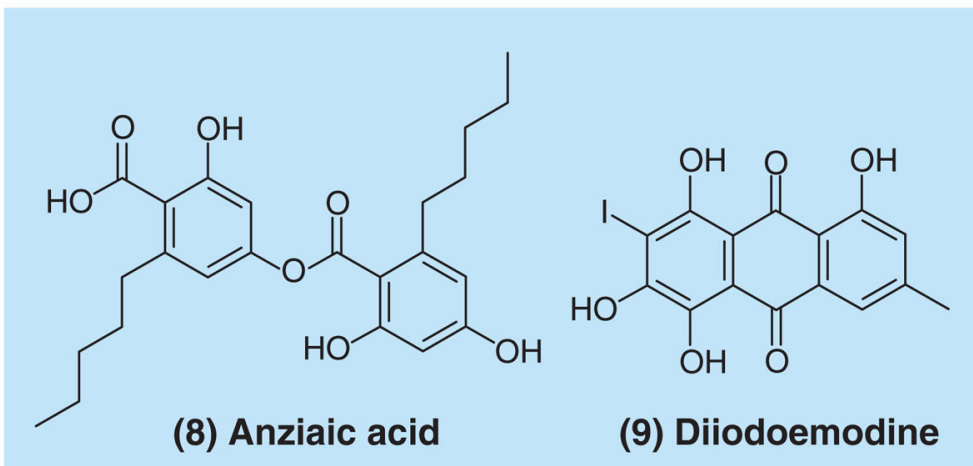

Figure 4.

Natural product derived compounds that can act as dual inhibitors of bacterial topoisomerase I and DNA gyrase. 


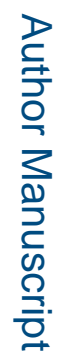

胥

颃
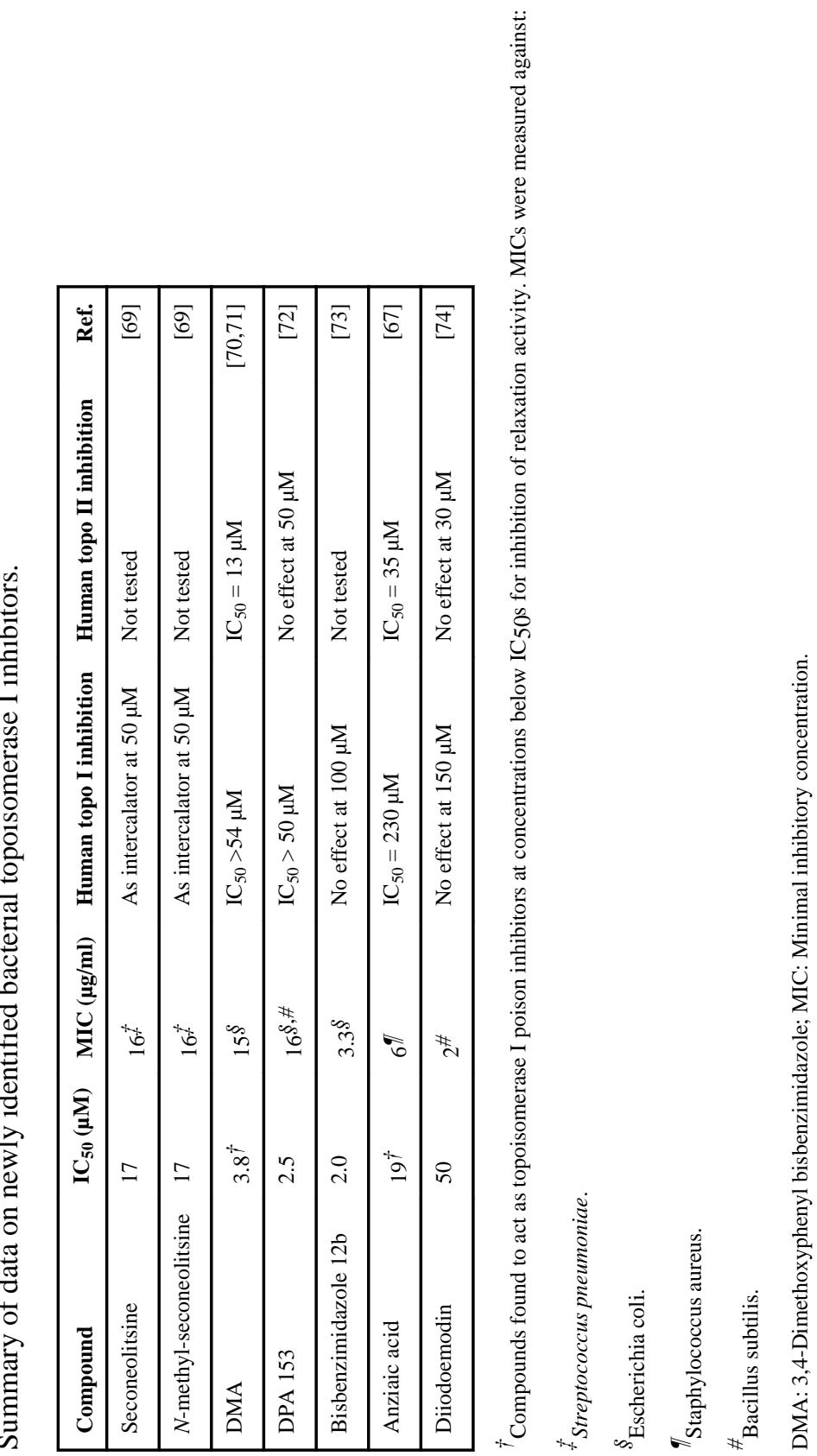

Future Med Chem. Author manuscript; available in PMC 2016 January 01. 